\title{
Frecuencia, susceptibilidad antimicrobiana y patrón de adherencia de Salmonella enterica aislada de carne de pollo, res y cerdo de la Ciudad de México
}

\author{
Sergio Villalpando-Guzmán, Carlos Ramón Vázquez-Quiñones, Iván Natividad-Bonifacio, \\ Everardo Curiel-Quesada, Elsa Irma Quiñones-Ramírez y Carlos Vázquez-Salinas
}

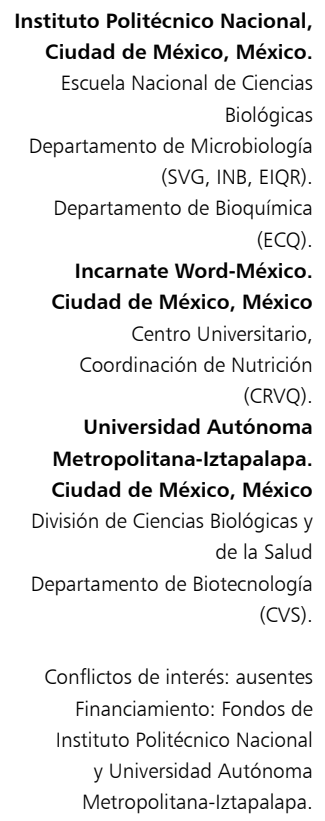

Conflictos de interés: ausentes Financiamiento: Fondos de Instituto Politécnico Nacional y Universidad Autónoma Metropolitana-Iztapalapa.

Recibido: 24 de enero de 2017 Aceptado: 22 de agosto de 2017

Correspondencia a: Carlos Vázquez-Salinas cvs@xanum.uam.mx

\section{Frequency, antimicrobial susceptibility and adherence patterns of Salmonella enterica isolated from chicken meat, beef and pork from Mexico City}

Background: Food of animal origin is often involved in salmonellosis outbreaks. Aim: To evaluate the frequency of Salmonella enterica in chicken, beef and pork ground meat (a total of 2,592 samples) obtained from travelling markets and supermarkets at the Iztapalapa area of Mexico City, in order to determine the antimicrobial susceptibility and adherence capacity of isolated strains. Methods: Isolation of S. enterica was carried out according to the BAM-FDA, the microbial susceptibility according with CLSI and adherence assay on HEp-2 cell line according with Baffone et al., 2001. Results: S. enterica was isolated from 511 of all the analyzed samples (19.7\%), from which $244(47.7 \%), 152(29.7 \%)$ and 115 (22.5\%) corresponded to chicken, beef and pork ground meat, respectively. The highest frequency of resistance of $S$. enterica to antimicrobials was to ampicillin and chloramphenicol in chicken, perfloxacin and ampicillin in beef and carbenicillin, ampicillin, chloramphenicol, cefotaxime and perfloxacin in pork. Ninety percent of the strains showed an aggregative adherence pattern on HEp- 2 cells. Conclusion: The frequency of $S$. enterica on meat products is high, which is the reason why a proper cooking of these ground meats is important in order to reduce the risk of acquiring salmonellosis.

Key words: Salmonella enterica, salmonellosis, chicken meat, beef, pork.

Palabras clave: Salmonella enterica, salmonelosis, carne de pollo, carne de res, carne de cerdo.

\section{Introducción}

L a salmonelosis continúa siendo un problema de salud pública en el mundo ${ }^{1}$. Actualmente se han descrito más de 2.500 serotipos de Salmonella, existiendo variaciones de tales serotipos en función de la región geográfica ${ }^{2}$. Este microorganismo está ampliamente distribuido en la naturaleza y puede sobrevivir en el ambiente por largos períodos ${ }^{1,3}$.

La presencia de Salmonella en los alimentos obedece a mecanismos de contaminación a través de la cadena de producción de los mismos, que involucra a las carnes, ingredientes como especias y la participación de portadores asintomáticos ${ }^{4,5}$. Dentro de los alimentos asociados con la salmonelosis se incluyen: carne de pollo, cerdo, res, huevo, productos lácteos y de origen marino, perejil, cilantro, brócoli, coliflor, lechuga y espinacas, entre otros ${ }^{6-8}$.

La contaminación cruzada de las carnes de pollo, res y cerdo ocurre principalmente por el contacto con superficies contaminadas como muebles, utensilios y equipo. Los productos listos para consumo, preparados con carnes y vegetales representan un riesgo a la población, ya que de ellos se ha aislado Salmonella .
En México, la SAGARPA-SIAP reportó un incremento en el volumen de la producción nacional de carne en canal, de bovino, porcino y aves, en el período de 2012 a $2014^{10}$; no obstante, también el volumen de importaciones de carnes de pollo, res y cerdo muestra una tendencia al alza ${ }^{11}$.

La selección de antimicrobianos para el tratamiento de salmonelosis, (cuando aquello es necesario), debe estar idealmente basado en el aislamiento de las cepas y la determinación de la susceptibilidad antimicrobiana. En la práctica, no siempre se hace de esta manera, ya que el tiempo que implica el análisis desde el muestreo hasta la obtención de resultados no es compatible con el tratamiento rápido que pudiera requerirse ${ }^{12}$. En el tema de los animales de granjas, así como de la carne obtenida de éstos, se ha reportado alrededor del mundo el aislamiento de cepas de Salmonella que son resistentes a diferentes antimicrobianos tales como penicilinas, cefalosporinas, aminoglucósidos, sulfonamidas y tetraciclinas ${ }^{13,14}$. Esta situación viene a complicar el tratamiento de salmonelosis en el ser humano ${ }^{15,16}$.

Desde tiempo de los aztecas, ha existido una cultura muy arraigada en la región por la compra - venta de productos en mercados, que actualmente conocemos como 
mercados sobre ruedas o "tianguis", que se caracterizan por ser puestos no fijos ubicados en las calles, sin ningún servicio sanitario, donde se expenden diferentes tipos de productos, entre ellos, alimentos crudos como carnes, frutas, hortalizas o bien, alimentos preparados y listos para su consumo, entre otras cosas (Figura 1). Debido al ritmo de vida actual, se ha incrementado la demanda en el consumo de comida rápida, como las hamburguesas o comidas típicas mexicanas a base de carnes molidas.

Lo anterior motivó evaluar la frecuencia de Salmonella enterica en carnes molidas de pollo, res y cerdo obtenidas de mercados sobre ruedas y supermercados de la delegación Iztapalapa en la Ciudad de México, determinar en estas cepas la susceptibilidad antimicrobiana in vitro y su capacidad de adherencia en células HEp-2.

\section{Materiales y Métodos}

\section{Recolección de muestras}

Las muestras fueron adquiridas en tres diferentes mercados sobre ruedas y tres diferentes supermercados en la delegación Iztapalapa perteneciente a la Ciudad de México. El período de muestreo fue de enero a diciembre de 2010. Se obtuvo un total de 2.592 muestras de carne molida de res, pollo y cerdo (864 de cada una). Setenta y dos muestras de cada tipo de carne molida fueron analizadas por mes, haciendo un total de 864 muestras de cada una por año. La cantidad de muestra recolectada fue de $1.000 \mathrm{~g}$, en cada caso, transportadas en hielera a $4{ }^{\circ} \mathrm{C}$ aproximadamente, el tiempo transcurrido entre su obtención y el análisis no fue mayor de dos horas.

\section{Aislamiento de Salmonella enterica}

El aislamiento e identificación de $S$. enterica se hizo de acuerdo a la metodología del BAM-FDA ${ }^{17}$. Se pesó $25 \mathrm{~g}$ de carne molida en $225 \mathrm{~mL}$ de caldo lactosado y se mezcló por 2 min. Luego se transfirió de manera aséptica la mezcla homogeneizada a un frasco estéril de boca ancha con tapón de rosca de $500 \mathrm{~mL}$ y se dejó reposar durante $60 \pm 5 \mathrm{~min}$ a temperatura ambiente, se ajustó el $\mathrm{pH}$ a $6,8 \pm 0,2$, se adicionó $2,25 \mathrm{~mL}$ de tergitol anionico 7 (sometido a vapor durante $15 \mathrm{~min}$ ) y se mezcló. Enseguida se incubó a $35^{\circ} \mathrm{C}$ por $24 \mathrm{~h}$, posteriormente se inoculó 0,1 y 1,0 $\mathrm{mL}$ del enriquecimiento en $10 \mathrm{~mL}$ de caldo Rapapport-Vassiliadis (RV) y caldo base tetrationato (TTB), respectivamente. El RV se incubó a $42 \pm 0,2{ }^{\circ} \mathrm{C} / 24$ h y el TTB se incubó a $43 \pm 0,2$ ${ }^{\circ} \mathrm{C} / 24$ h, se aisló en agares selectivos y se incubó a 35 ${ }^{\circ} \mathrm{C} / 24 \mathrm{~h}$. Dos colonias con características de Salmonella fueron elegidas de cada medio de cultivo y se hizo la identificación bioquímica y la confirmación serológica con antisuero polivalente. Las cepas confirmadas como Salmonella fueron enviadas al Instituto de Diagnóstico

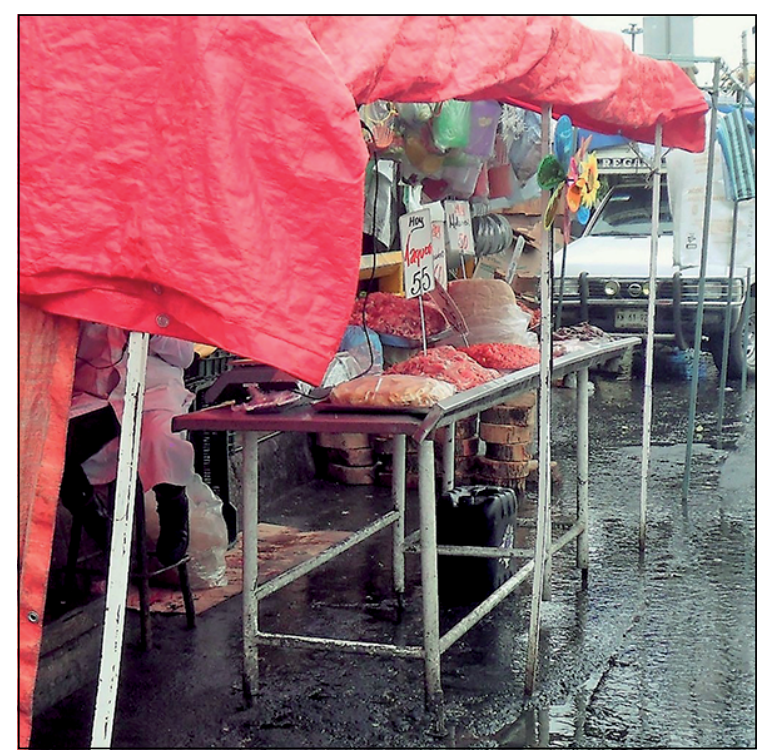

Figura 1. Local típico de un mercado sobre ruedas en la Ciudad de México donde se venden diferentes tipos de carnes molidas. y Referencia Epidemiológicos de la Secretaría de Salud de México para la identificación de serotipo.

\section{Prueba de susceptibilidad antimicrobiana}

Esta prueba fue llevada a cabo de acuerdo con el Instituto de Estándares Clínicos y de Laboratorios (CLSI, por su sigla en inglés $)^{18}$. Las cepas de Salmonella se crecieron en caldo infusión cerebro-corazón (BHI), se ajustó la turbiedad del caldo a 0,5 del nefelómetro de McFarland y se sembró en agar Mueller Hinton; el pH se ajustó a 7,3 $\pm 0,1$. Los multidiscos usados fueron de la marca Biorad cat.71080280. Se empleó la cepa de Escherichia coli ATCC 25922 como control, ya que el $\mathrm{CLSI}^{18}$ describe los intervalos en $\mathrm{mm}$ de los halos de inhibición que esta cepa desarrolla para cada uno de los antimicrobianos probados, los que deben ser reproducidos por cada laboratorio. La interpretación de resultados fue de acuerdo al documento M45-A2 ${ }^{18}$.

\section{Ensayo de adherencia en células HEp-2}

Se prepararon microplacas de 24 pozos a $100 \%$ de confluencia de células HEp-2 (línea celular obtenida de cáncer de células laríngeas). Las células se crecieron sobre portaobjetos estériles, se adicionaron $975 \mu \mathrm{L}$ de medio mínimo esencial (MEM) más $25 \mu \mathrm{L}$ de la suspensión bacteriana ajustadas al tubo 0,5 del nefelómetro de MacFarland por pozo. Y luego, se incubaron las microplacas a $37{ }^{\circ} \mathrm{C}$ con $5 \%$ de atmósfera de $\mathrm{CO}_{2}$ durante $1,5 \mathrm{~h}$. El medio fue desechado con pipeta Pasteur y se lavaron las microplacas tres veces con solución reguladora de fosfatos estéril (PBS), se fijaron las células con metanol durante 1 min y se lavaron tres veces con PBS estéril. Después, 
las células fueron teñidas con Giemsa durante $20 \mathrm{~min}$; los pozos fueron lavados con agua destilada deshidratados con acetona-xilol y selladas con una gota de resina de Permount. Se observaron las preparaciones en microscopio con el objetivo de inmersión (100x). Los ensayos de adherencia fueron considerados positivos cuando más de $40 \%$ de las células mostraron adherencia bacteriana ${ }^{19}$.

\section{Análisis de datos}

En el análisis de los resultados se utilizó el programa PAST Paleontological Statistics Software Package for Education and Data Analysis v. 3.10 University of Oslo ${ }^{20}$ para determinar diferencias significativas por tipo de muestras y sitio de muestreo.

\section{Resultados}

\section{Aislamiento de Salmonella enterica}

Del total de muestras de carne molida analizadas, en $19,7 \%(511 / 2.592)$ se detectó $S$. enterica. De las muestras positivas $47,7 \%(244 / 511)$ fueron de pollo, $29,7 \%$ $(152 / 511)$ de res y $22,5 \%(115 / 511)$ de cerdo.

\section{Procedencia de las muestras positivas}

De las muestras positivas de pollo, $62,3 \%$ provenían de mercados sobre ruedas y $37,7 \%$ de supermercados. De las muestras positivas de carne de res, $63,1 \%$ fueron de mercados sobre ruedas y $36,8 \%$ de los supermercados.
En la carne de cerdo, 62,6\% fueron de mercados sobre ruedas y $37,3 \%$ de supermercados. De las 511 muestras positivas, $320(62,6 \%)$ correspondieron a las muestras recolectadas en los mercados sobre ruedas y $191(37,3 \%)$ a supermercados. De acuerdo a los datos obtenidos, en la carne de pollo se obtuvo el mayor número de muestras positivas para $S$. enterica, seguida de la res y por último la de cerdo.

\section{Temporalidad de los aislamientos}

En la Figura 2 se muestra el total de muestras positivas (suma de muestras positivas de carne de pollo, res y cerdo) para el aislamiento de $S$. enterica de los tres mercados sobre ruedas o supermercados, por mes. El aislamiento de $S$. enterica se presentó prácticamente durante todo el período de muestreo, teniendo el mayor número de muestras positivas de mayo a septiembre, tanto en mercados sobre ruedas como en supermercados.

El análisis estadístico de las muestras que resultaron positivas para el aislamiento de $S$. enterica, demostró una mayor probabilidad de aislamiento de $S$. enterica en carne molida de pollo comparada con la de res (odd ratio $=1,843 ; \mathrm{IC}=1,466-2,319)$ y la de cerdo (odd ratio $=2,563 ; \mathrm{IC}=2,004-3,278)$, en tanto que la frecuencia de aislamiento de $S$. enterica entre las carnes molidas de res y cerdo es menor (odd ratio $=1,390$; IC $=1,069-1,809$ ).

Existen diferencias significativas $(\mathrm{p}<0,05)$ del aislamiento de $S$. enterica entre las muestras de carne molida
Figura 2. Muestras positivas del aislamiento de Salmonella enterica a partir de carnes molidas de pollo, res y cerdo durante un año.

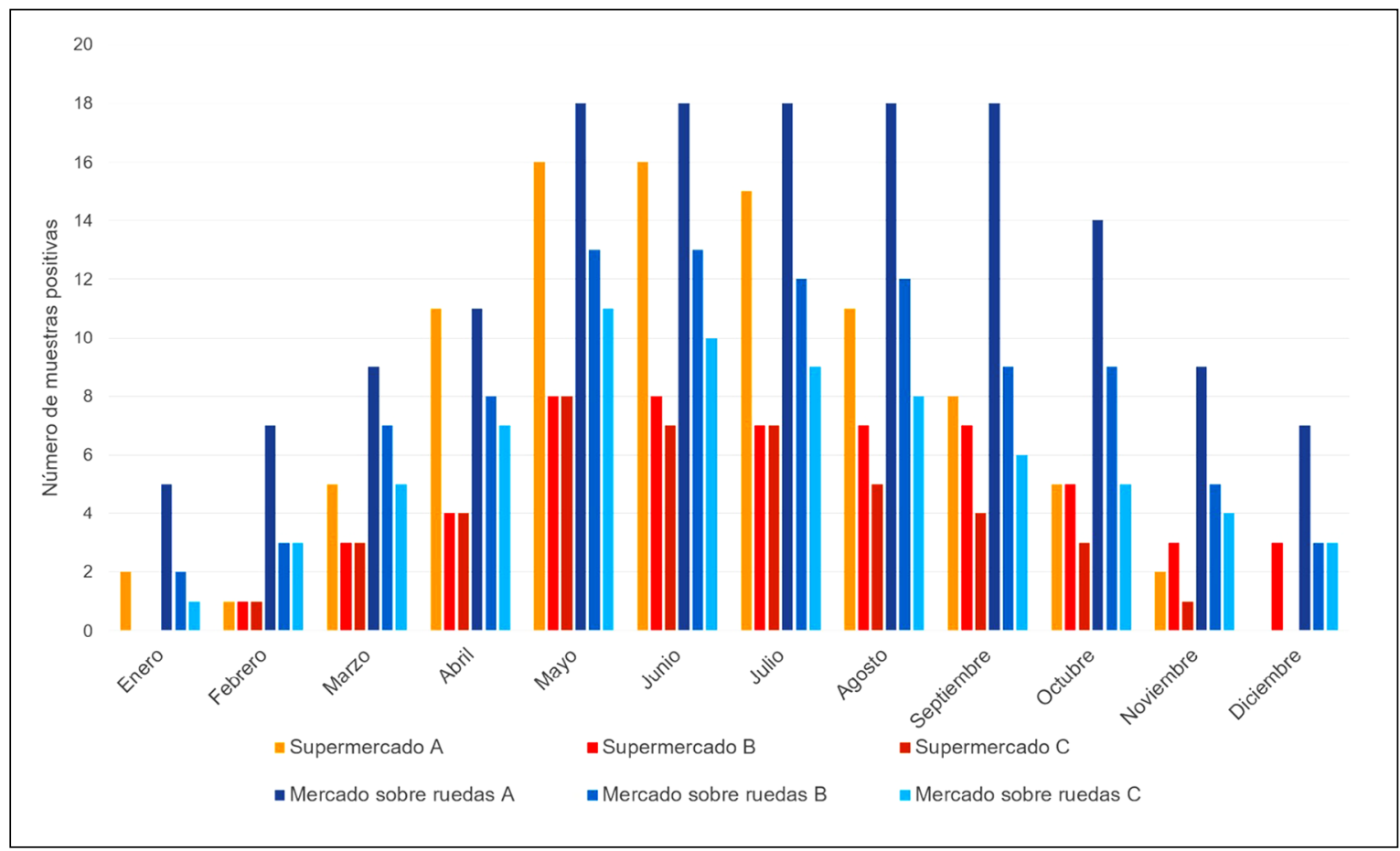


de pollo, res y cerdo obtenidas de mercados sobre ruedas y supermercados, siendo más frecuente el aislamiento de $S$. enterica en muestras obtenidas de mercados sobre ruedas para los tres tipos de carnes molidas.

\section{Serotipos de Salmonella identificados}

Del total de muestras que resultaron positivas para el aislamiento de $S$. enterica (511), 358 cepas fueron serotipificadas. Conforme se presenta en la Tabla 1, los serotipos más frecuentes fueron Salmonella enterica ser. Anatum, Salmonella enterica ser. Newport y Salmonella enterica ser. Typhimurium (Tabla 1). Un total de 153 cepas fueron no tipificables.

\section{Susceptibilidad antimicrobiana}

Noventa y cinco por ciento (132/139) de las cepas aisladas de carne molida de pollo fueron resistentes a ampicilina $10 \mu \mathrm{g}$, seguido de cloranfenicol $30 \mu \mathrm{g}$ con $82 \%$ de resistencia (114/139). En carne molida de res, 92,4\% (109/118) fueron resistentes a perfloxacina $5 \mu \mathrm{g}$ y $92,4 \%$ lo fueron a ampicilina $10 \mu \mathrm{g}$. El 100\% de las cepas aisladas de carne molida de cerdo (101/101) fueron resistentes a

\begin{tabular}{|c|c|c|c|c|c|c|c|c|c|c|c|c|c|c|c|c|}
\hline $\begin{array}{l}\text { Tipo de } \\
\text { muestra }\end{array}$ & $\begin{array}{c}\mathrm{n} \text { de } \\
\text { muestras }\end{array}$ & $\begin{array}{l}\text { Muestras } \\
\text { positivas }\end{array}$ & $\begin{array}{c}\text { Serotipos } \\
\text { identificados }\end{array}$ & $\begin{array}{l}\mathrm{n} \text { de cepas } \\
\text { por serotipo }\end{array}$ & AK & CRO & $\mathrm{CF}$ & $\mathrm{CB}$ & AM & $\mathrm{CL}$ & GE & CTX & STX & NF & NET & PEF \\
\hline \multirow[t]{7}{*}{ Pollo } & 864 & 244 & $\begin{array}{l}\text { Salmonella enterica } \\
\text { ser. Anatum }\end{array}$ & 27 & 1 & 1 & 1 & 27 & 27 & 27 & 7 & 27 & 2 & 1 & 1 & 27 \\
\hline & & & $\begin{array}{l}\text { Salmonella enterica } \\
\text { ser. Newport }\end{array}$ & 16 & 1 & 2 & 2 & 16 & 16 & 16 & 4 & 16 & 2 & 1 & 2 & 16 \\
\hline & & & $\begin{array}{l}\text { Salmonella enterica } \\
\text { ser. Typhimurium }\end{array}$ & 15 & 2 & 2 & 1 & 15 & 15 & 15 & 2 & 15 & 1 & 1 & 1 & 15 \\
\hline & & & $\begin{array}{l}\text { Salmonella enterica } \\
\text { ser. Gallinarum }\end{array}$ & 46 & 1 & 1 & 1 & 13 & 43 & 30 & 13 & 19 & 1 & 2 & 1 & 10 \\
\hline & & & $\begin{array}{l}\text { Salmonella enterica } \\
\text { ser. Derby }\end{array}$ & 4 & 1 & 2 & 1 & 4 & 4 & 4 & 2 & 4 & 1 & 1 & 1 & 4 \\
\hline & & & $\begin{array}{l}\text { Salmonella enterica } \\
\text { ser. Pullorum }\end{array}$ & 31 & 1 & 2 & 2 & 7 & 27 & 22 & 9 & 21 & 1 & 2 & 1 & 15 \\
\hline & & & $\begin{array}{l}\text { Porcentaje de } \\
\text { resistencia }\end{array}$ & & 5 & 7,2 & 5,8 & 59,0 & 95,0 & 82,0 & 26,6 & 73,4 & 5,8 & 5,8 & 5,0 & 62,6 \\
\hline \multirow[t]{5}{*}{ Res } & 864 & 152 & $\begin{array}{l}\text { Salmonella enterica } \\
\text { ser. Anatum }\end{array}$ & 48 & 3 & 2 & 1 & 27 & 39 & 37 & 2 & 23 & 2 & 3 & 2 & 48 \\
\hline & & & $\begin{array}{l}\text { Salmonella enterica } \\
\text { ser. Newport }\end{array}$ & 22 & 2 & 10 & 4 & 22 & 22 & 22 & 3 & 22 & 1 & 2 & 2 & 22 \\
\hline & & & $\begin{array}{l}\text { Salmonella enterica } \\
\text { ser. Typhimurium }\end{array}$ & 34 & 3 & 5 & 2 & 34 & 34 & 34 & 1 & 34 & 2 & 4 & 2 & 32 \\
\hline & & & $\begin{array}{l}\text { Salmonella enterica } \\
\text { ser. Derby }\end{array}$ & 14 & 1 & 3 & 1 & 14 & 14 & 14 & 3 & 14 & 2 & 2 & 1 & 13 \\
\hline & & & $\begin{array}{l}\text { Porcentaje de } \\
\text { resistencia }\end{array}$ & & 7,6 & 16,9 & 6,8 & 82,2 & 92,4 & 90,7 & 7,6 & 78,8 & 5,9 & 9,3 & 5,9 & 97,5 \\
\hline \multirow[t]{5}{*}{ Cerdo } & 864 & 115 & $\begin{array}{l}\text { Salmonella enterica } \\
\text { ser. Anatum }\end{array}$ & 32 & 3 & 4 & 2 & 32 & 32 & 32 & 2 & 32 & 1 & 1 & 0 & 32 \\
\hline & & & $\begin{array}{l}\text { Salmonella enterica } \\
\text { ser. Newport }\end{array}$ & 37 & 1 & 2 & 1 & 37 & 37 & 37 & 2 & 37 & 2 & 3 & 2 & 37 \\
\hline & & & $\begin{array}{l}\text { Salmonella enterica } \\
\text { ser. Typhimurium }\end{array}$ & 22 & 2 & 3 & 2 & 22 & 22 & 22 & 1 & 22 & 3 & 3 & 2 & 22 \\
\hline & & & $\begin{array}{l}\text { Salmonella enterica } \\
\text { ser. Derby }\end{array}$ & 10 & 1 & 1 & 2 & 10 & 10 & 10 & 2 & 10 & 2 & 3 & 1 & 10 \\
\hline & & & $\begin{array}{l}\text { Porcentaje de } \\
\text { resistencia }\end{array}$ & & 6,9 & 9,9 & 6,9 & 100 & 100 & 10 & 6,9 & 100,0 & 7,9 & 9,9 & 5,0 & 100,0 \\
\hline
\end{tabular}

Amikacina (AK) $30 \mu \mathrm{g}$; Ceftriaxona (CRO) $30 \mu \mathrm{g}$; Cefalotina (CF) $30 \mu$; Carbenicilina (CB) $100 \mu \mathrm{g}$; Ampicilina (AM) $10 \mu \mathrm{g}$; Cloramfenicol (CL) $30 \mu \mathrm{g}$; Gentamicina (GE) 10 $\mu \mathrm{g}$; Cefotaxima (CTX) $30 \mu \mathrm{g}$; Cotrimoxazol (STX); Nitrofurantoina (NF) $300 \mu \mathrm{g}$; Netilmicina (NET) $30 \mu \mathrm{g}$; Perfloxacina (PEF) $5 \mu \mathrm{g}$ 


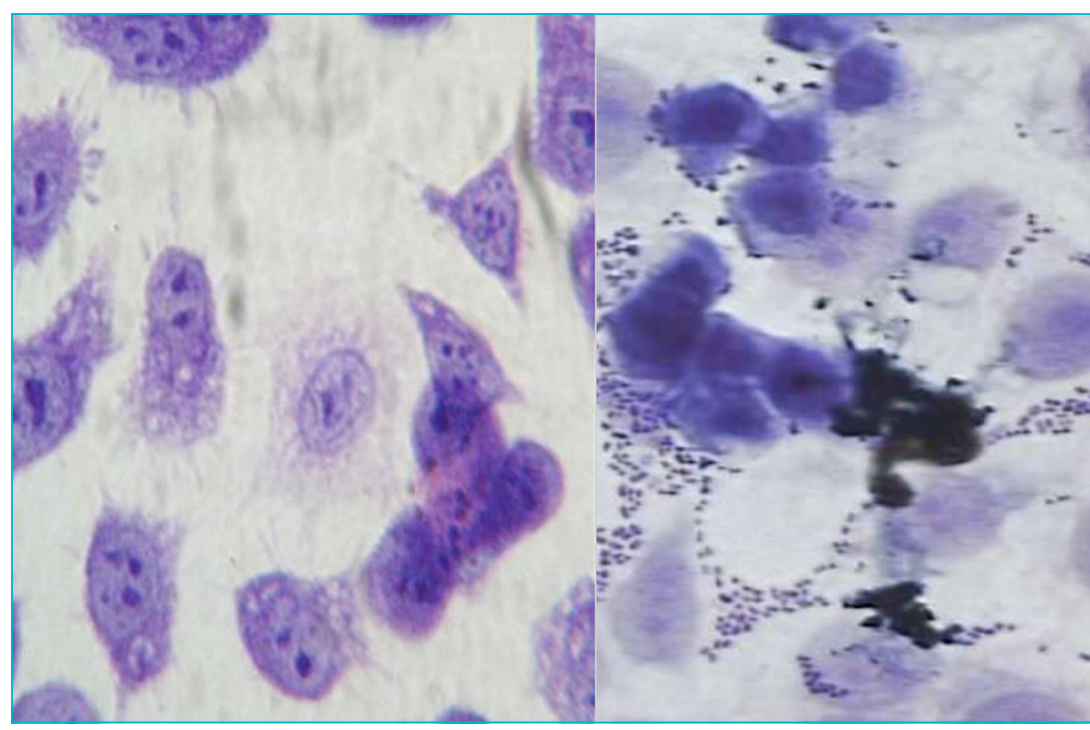

Figura 3. Patrón de adherencia agregativo de Salmonella en células HEp-2 (derecha) y control negativo (izquierda).
- En Taiwán, se ha comunicado una frecuencia de $30 \%$, reportando los serotipos $S$. enterica ser. Albany $(36,7 \%)$, Schwarzengrund (33,5\%), Istanbul $(13,2 \%)$, Derby $(6,9 \%)$, Typhimurium $(3 \%)$, Anatum $(0,8 \%)$, Haardt (0,8\%), Virchow $(0,8 \%)$, Senftenberg $(0,6 \%)$, $\mathrm{y}$ otros serotipos que en conjunto representaron un $2,8 \%$ de frecuencia ${ }^{23}$.

- En Australia, se encontró en 38,8\%, donde el principal serotipo fue $S$. enterica ser. Typhimurium $(61,7 \%)^{24}$

- En Egipto, se reportó 44\%, específicamente del serotipo $S$. enterica ser. Typhimurium ${ }^{25}$.

- En Guatemala, 34,3\%, siendo los serotipos más frecuentes $S$. enterica ser. Paratyphi B (34,8\%), Heidelberg $(16,3 \%)$ y Derby $(11,6 \%)^{27}$.

En el presente estudio, la frecuencia de aislamiento de $S$. enterica en carne molida de pollo fue de $47,7 \%$, porcentaje que es superior a los reportado en los países anteriores. Los serotipos con mayor frecuencia (Tabla $1)$ fueron $S$. enterica ser. Gallinarum $(18,8 \%)$ y Pullorum $(12,7 \%)$, los que son de importancia veterinaria, seguido de $S$. enterica ser. Anatum (11\%), Newport $(6,5 \%)$, Typhimurium $(6,1 \%)$ y Derby $(1,6 \%)$. Este patrón de serotipos es diferente a lo reportando en los países antes citados, existiendo algunas coincidencias como el caso de $S$. enterica ser. Anatum, Derby y Typhimurium, aunque con diferencias en la frecuencia de aislamiento. El serotipo Typhimurium es uno de los más ampliamente distribuidos en el mundo, con una variedad de hospederos que incluyen al ser humano, pollos, cerdos, terneras, entre otros ${ }^{48,49}$ y es reconocido junto a los serotipos Enteritidis, Newport y Heidelberg como los principales serotipos patógenos transmitidos por alimentos en el humano ${ }^{26}$.

La diversidad de serotipos encontrados en los diferentes países y el cambio en la frecuencia de serotipos es un reflejo de la influencia del comercio internacional y compra-venta de animales y alimentos de origen animal ${ }^{50}$.

En la carne de res, nuestra investigación mostró una frecuencia de aislamiento de $S$. enterica de 29,7\%, siendo los serotipos encontrados Anatum, Typhimurium, Newport y Derby con una frecuencia de 31, 22, 14 y 9\%, respectivamente.

En otras latitudes se ha comunicado las siguientes frecuencias de contaminación de carne de res por S. enterica:

- En Egipto ${ }^{26}$ se reportó $23,3 \%$ de frecuencia, de las cuales, 38,2 - 34,6 - 11 - 7 - 4,4 y 2,6\%, correspondieron con los serotipos Typhimurium, Enteritidis, Haifa, Muenster, Virchow y Anatum, respectivamente.

- En Etiopía ${ }^{28}$ se encontró 7,07\% de frecuencia siendo los serotipos predominantes Mishmarhaemek, Typhimurium, Newport, Eastbourne, Infantis, Anatum, con 20 - 17,4 - 13 - 8,7 - 7,3 y 5,8\% de frecuencia, respectivamente. 
- En Irlanda ${ }^{29}$ se reportan frecuencias de $0,16 \%$, siendo los serotipos Typhimurium, Dublin y Kentucky los más frecuentes con 26-24 y 10\%, respectivamente.

- En el Reino Unido ${ }^{30}$ se encontró una frecuencia de 1,1\%, siendo los serotipos más comunes Typhimurium y Derby con 54,2 y $16,9 \%$, respectivamente.

De la misma forma, el porcentaje de aislamiento de Salmonella enterica de nuestro trabajo resultó superior en comparación con los países mencionados, siendo el serotipo Typhimurium muy común en la carne molida de res.

En carne de cerdo, en nuestro trabajo encontramos una frecuencia de $22,5 \%$. En otros países ha resultado mayor, por ejemplo:

- En Irlanda $40 \%{ }^{31}$, siendo los serotipos predominantes Typhimurium y Derby con 52 y $35 \%$, respectivamente.

- En Etiopía, la frecuencia ha sido de 43,8\%; los serotipos Hadar, Eastbourne, Saintpaul, Kentucky y Typhimurium fueron los más frecuentes con $31,8-15$, $13-7,5$ y $5,6 \%$, respectivamente.

- En China ${ }^{51}$, se reportó 29,2\% de frecuencia, y nuevamente aparecen los serotipos Typhimurium, Derby y Enteritidis como los predominantes: 28 - 27 y $8,6 \%$, respectivamente.

Para carne de cerdo, los serotipos coincidentes entre los países indicados y nuestro trabajo resultaron Typhimurium y Derby.

La única información que se tiene en México en relación los serotipos de $S$. enterica aislada de alimentos es del Instituto de Diagnóstico y Referencia Epidemiológico $^{39}$ que reportó durante tres décadas (1972-1999), que los serotipos más frecuentemente aislados en alimentos fueron Derby, Anatum, Agona, Typhimurium y Enteritidis. El 51\% correspondió a alimentos preparados, 23\% a cárnicos (productos derivados de la carne como: jamón, longaniza, chorizo, queso de puerco, etc.), $22 \%$ a carne cruda (molida de res, pollo, pescado), $3 \%$ a lácteos y $1 \%$ a huevo (fresco y en polvo).

La contaminación con Salmonella de las canales, piezas separadas y vísceras de pollo son frecuentemente reportados alrededor del mundo ${ }^{26,32}$. Algunos investigadores ${ }^{33}$ destacan la importancia que tiene Salmonella como biota frecuente de los pollos en granjas, rastros, carne molida de pollo o en piezas en plantas procesadoras en E.U.A.

La molienda es un factor adicional que puede contaminar cualquier tipo de carne. La contaminación de la carne en trozos o en filetes puede ocurrir sólo en la superficie, en tanto el interior se mantiene estéril. La molienda incorpora bacterias desde la superficie al interior de la carne; además se propicia un ambiente de micro-aerofilia propicio para la multiplicación de muchas bacterias. La contaminación cruzada durante la molienda ocurre cuando se mezclan piezas de carne contaminadas con las que no lo están. Adicionalmente, los residuos de carne que permanecen en un molino no sanitizado permiten la proliferación bacteriana $^{34,35}$.

En un estudio realizado en Jalisco, México, se comparó la frecuencia del aislamiento de Salmonella en canales de res, trozos de carne de res y carne molida de res de carnicerías, encontrándose un mayor porcentaje de aislamiento en la carne molida de res, seguido de los trozos y finalmente en las canales $(71,39$ y $18 \%$, respectivamente); este estudio evidenció mayores concentraciones de Salmonella en la carne molida de res en comparación con trozos de carne, ya que reportaron como valores promedio $2,3 \pm 1,1 \log \mathrm{NMP} / 25$ g y $1,9 \pm 0,9 \log \mathrm{NMP} / 25 \mathrm{~g}$, respectivamente ${ }^{36}$.

El riesgo de transmisión de Salmonella en las carnes molidas se incrementa cuando se consume cruda, ahumada o parcialmente cocida ${ }^{37}$. Actualmente, el consumo de hamburguesas de pollo o res o hamburguesas hechas con la mezcla de carnes de res y cerdo, parcialmente cocidas, es muy común como parte de la cultura de comida rápida, lo que representa un factor de riego de exposición del consumidor a Salmonella. En nuestros resultados, la mayor frecuencia de aislamiento de $S$. enterica fue en la carne molida de pollo, seguida de la carne molida de res y la carne molida de cerdo. La salmonelosis afecta a todos los grupos de edades; sin embargo, la mayor frecuencia de casos se concentra en el grupo de 25 a 44 años de edad, correspondiente a la mayor parte de la población económicamente activa, de manera que la enfermedad tiene un alto impacto socio-económico, por lo que es importante aplicar medidas preventivas para su control ${ }^{38}$.

Durante el período de muestreo, fue evidente la mayor frecuencia del aislamiento de $S$. enterica en los meses cálidos (primavera-verano) tanto en mercados sobre ruedas como en supermercados para los tres tipos de carnes molidas (Figura 2).

En nuestra investigación, se evidenció la resistencia de $S$ - enterica a varios antimicrobianos; sin embargo, no se observó relación de esta resistencia con los serotipos. Existen reportes que demuestran alta variabilidad en la susceptibilidad a antimicrobianos de las cepas de Salmonella. Por ejemplo, cepas de Salmonella aisladas de terneras en Australia ${ }^{12}$ son resistentes a estreptomicina (25\%), ampicilina (18\%) o combinación de sulfonamidas (21\%); otros investigadores reportaron que $48 \%$ de las cepas de Salmonella aisladas de pollos, cerdos y vacas lecheras de granjas en $\mathrm{China}^{13}$, mostraron resistencia a por lo menos un antimicrobiano, siendo más frecuente a cotrimoxazol (39\%), ácido nalidíxico (39\%), doxiciclina (28\%) y tetraciclina (27\%). También, Sibhat y cols. ${ }^{40}$, reportaron que $20 \%$ de las cepas de Salmonella aisladas en carne de res en Etiopía fueron resistentes a uno o más antimicrobianos, principalmente estreptomicina, sulfisoxazol 
y tetraciclina. Yangkam-Yhiler y cols. ${ }^{14}$, reportaron que $86 \%$ de las cepas de Salmonella aisladas de pollos de granjas y de rastros en Nigeria mostraron resistencia a uno o más antimicrobianos, siendo predominante la resistencia a ampicilina $(72,9 \%)$.

En México ${ }^{41}$, ampicilina está aprobada para administración en bovinos, cerdo y aves de corral; sin embargo, esta práctica evidenció alta resistencia a ampicilina de las cepas de $S$. enterica aisladas en el presente estudio de los tres tipos de carnes $(95 \%$ en pollo, $92,4 \%$ en res y $100 \%$ en cerdo), lo que es mayor a lo reportado en otros países. Nuestra investigación también demostró la resistencia de cepas de $S$. enterica a cefalosporinas tales como cefotaxima en el caso de carne de cerdo y a quinolonas tales como pefloxacina en el caso de res y cerdo, no obstante que estos antimicrobianos no están autorizados para uso veterinario en México ${ }^{41}$. También se evidenció la resistencia a cloranfenicol en cepas de $S$. enterica aisladas de pollo y cerdo, a pesar de que este antimicrobiano está prohibido en cualquier animal destinado al consumo humano ${ }^{41}$.

Otra característica evaluada en las cepas de $S$. enterica aisladas de los tres diferentes tipos de carnes fue la capacidad de adherencia "in vitro" en células HEp-2. La capacidad de adherencia a células hospederas es crítica para un microorganismo patógeno cause enfermedad. El efecto "in vivo" a nivel intestinal es muy semejante a lo observado "in vitro" con células HEp-2, ya que estas células re-arreglan sus filamentos de actina facilitando la penetración inducida por Salmonella, en forma similar a como ocurre en el hospedero infectado ${ }^{42}$ y porque la bacteria reconoce los receptores de manosa a los que se adhiere de la misma forma que en los enterocitos ${ }^{43}$. Este proceso, de manera semejante a otras enterobacterias como sucede con Escherichia coli enteropatógena, permite la acumulación de la actina del citoesqueleto en la región apical de la célula, hasta formar una estructura de tipo "pedestal" y causar la eliminación de las microvellosidades intestinales ${ }^{44}$. Althouse y cols. ${ }^{45}$, demostraron la capacidad de adherencia de Salmonella ser. Typhimurium aislada de cerdos, en las que se involucran fimbrias tipo I, en tanto que células mutantes de Salmonella no adherentes no tienen la capacidad de colonizar los enterocitos de cerdo. Ledeboer y cols. ${ }^{46}$, demostraron la capacidad de adherencia en células HEp-2 de Salmonella ser. Typhimurium patógenas aisladas de pollos y terneras, a través de la expresión de fimbrias tipo I. También se ha descrito la capacidad de adherencia de diferentes serotipos de Salmonella en células HEp-2 como consecuencia de la expresión de adhesinas (Fim $\mathrm{H}$ ) que forman parte de las fimbrias tipo I, las que pueden variar estructuralmente de una cepa a otra dentro de un mismo serovar. Esta adherencia fue mayor en cepas de Salmonella ser. Typhimurium y Salmonella ser. Newport; sin embargo, otros serotipos tales como Salmonella ser. Anatum y Salmonella ser. Pullorum también mostraron esta capacidad, aunque el número de células adheridas fue menor ${ }^{47}$. En nuestro estudio, se demostró que $90 \%$ (460/511) de las cepas de $S$. enterica aisladas de los tres tipos de carnes molidas tienen la capacidad de adherencia agregativa en células HEp-2, lo que representa un potencial de patogenicidad importante.

\section{Conclusiones}

Del total de muestras de carne molida analizadas en $19,7 \%(511 / 2.592)$ se detectó $S$. enterica. De las muestras positivas $47,7 \%$ fueron de pollo, $29,7 \%$ de res y $22,5 \%$ de cerdo, por lo que es importante la adecuada cocción de la carne en cualquiera de sus presentaciones; asimismo, evitar la contaminación cruzada para disminuir el riesgo de adquirir una salmonelosis de origen zoonótico.

\section{Resumen}

Introducción: Los alimentos de origen animal frecuentemente están implicados en brotes de salmonelosis. Objetivo: Evaluar la frecuencia de Salmonella enterica en carnes molidas de pollo, res y cerdo (un total de 2.592 muestras) obtenidas de mercados sobre ruedas y supermercados de la Delegación Iztapalapa en la Ciudad de México, determinar la susceptibilidad antimicrobiana y efectuar ensayos de adherencia en las cepas aisladas. Métodos: El aislamiento de S. enterica se hizo de acuerdo a la BAM-FDA, la susceptibilidad antimicrobiana de acuerdo con CLSI y el ensayo de adherencia en células HEp-2 conforme a Baffone y cols., 2001. Resultados: Salmonella enterica fue aislada en 511 del total de muestras analizadas (19,7\%), de las cuales 244 (47,7\%), $152(29,7 \%)$ y $115(22,5 \%)$ correspondieron a carne molida de pollo, res y cerdo, respectivamente. La mayor frecuencia de resistencia de $S$. enterica a antimicrobianos fue a ampicilina y cloranfenicol en pollo, perfloxacina y ampicilina en res y carbenicilina, ampicilina, cloranfenicol, cefotaxima y perfloxacina en cerdo. Noventa por ciento de las cepas mostraron un patrón de adherencia agregativo. Conclusión: La frecuencia de S. enterica en productos cárnicos es alta, por lo que es importante la adecuada cocción de la carne para disminuir el riesgo de una salmonelosis. 


\section{Referencias bibliográficas}

1.- Abatcha M G, Zakaria Z, Kaur D G, Thong K L. Review Article: A trends of Salmonella and antibiotic resistance. Adv Life Sci Technol 2014; 17: 9-21.

2.- Pui C F, Wong W C, Chai L C, Tunung R, Jeyaletchumi P, Noor-Hidayah M S, et al. Salmonella: A foodborne pathogen. Int Food Res J 2011; 18: 465-73.

3.- Winfield M D, Groisman E A. Role of nonhost environments in the lifestyles of Salmonella and Escherichia coli. Appl Environ Microbiol 2003; 69: 3687-94.

4.- Bosilevac J M, Arthur T M, Bono J L, BrichtaHarhay D M, Kalchayanand N, King D A, et al. Prevalence and enumeration of Escherichia coli O157:H7 and Salmonella in U. S. abattoirs that process fewer than 1,000 head of cattle per day. J Food Prot 2009; 72: 1272-8.

5.- CDC. Salmonella Montevideo infections associated with salami products made with contaminated imported black and red pepperUnited States, July 2009-April 2010. MMWR Morb Mortal Wkly Rep 2010; 59: 1647-50.

6.- Lapidot A, Yaron M. Transfer of Salmonella enterica serovar Typhimurium from contaminated irrigation water to parsley in dependent on curli and cellulose, the biofilm matrix components. J Food Prot 2009; 3: 618 23.

7.- Pires S M, Vieira A R, Hald T, Cole D. Source attribution of human salmonellosis: An overview of methods and estimates. Foodborne Pathog Dis 2014; 11: 667-76.

8.- Abakpa G O, Umoh V J, Ameh J B, Yakubu S E, Kwaga J K P, Kamaruzaman S. Diversity and antimicrobial resistance of Salmonella enterica isolated from fresh produce and environmental samples. Environ Nanotech Monit Manag 2015; 3: 38-46.

9.- Gibbons I S, Adesiyun A, Seepersadsingh N, Rahaman S. Investigation for possible source(s) of contamination of ready-to-eat meat products with Listeria spp. and other pathogens in a meat processing plant in Trinidad. Food Microbiol 2006; 23: 359-66.

10.- Secretaría de Agricultura, Ganadería, Desarrollo Rural, Pesca y AlimentaciónServicio de Información Agroalimentaria y Pesquer (SAGARPA-SIAP). Resumen Nacional de Producción, precio, valor, animales sacrificados y peso 2014. http://www.siap.gob. $\mathrm{mx} /$ resumen-nacional-pecuario/ (Accedido el 18 de febrero de 2016)

11.- Secretaría de Agricultura, Ganadería, Desarrollo Rural, Pesca y Alimentación (SAGARPA). Estadísticas: Consumos Nacionales Aparentes 1990-2005. http:// www.sagarpa.gob.mx/Ganaderia/Estadisticas/ Paginas/default.aspx. (Accedido el 18 de febrero de 2016)
12.- Izzo, M M, Mohler V L, House J K. Antimicrobial susceptibility of Salmonella isolates recovered from calves with diarrhoea in Australia. Aust Vet J 2011; 89: 402-8.

13.- Kuang X, Hao H, Dai M, Wang Y, Ahmad I, Liu Z, et al. Serotypes and antimicrobial susceptibility of Salmonella spp. isolated from farm animals in China. Front Microbiol 2015; 6: 602

14.- Yangkam-Yhiler N, Enya-Bassey B Antimicrobial susceptibility patterns of Salmonella species from sources in poultry production settings in Calabar, Cross River State, Nigeria. Am J Health Res 2015; 3: 76-81.

15.- Errecalde J O. Uso de antimicrobianos en animales de consumo. Roma, Organización de las Naciones Unidas para la Agricultura y la Alimentación; 2004, p. 18-9.

16.- McEwen S A, Fedorka-Cray P J. Antimicrobial use and resistance in animals. Clin Infect Dis 2002; 34: 93-106.

17.- Food and Drug Administration-Bacteriological Analytical Manual (FDA-BAM). Chapter 5: Salmonella. http://www.fda.gov/Food/ FoodScienceResearch/LaboratoryMethods/ ucm070149.htm (Accedido el 07 de julio de 2017)

18.- Clinical and Laboratory Standards Institute (CLSI). Methods for Dilution Antimicrobia Susceptibility Tests for Bacteria That Grow Aerobically Approved Standard, $2^{\text {th }}$ ed. Document M45-A2. Pennsylvania, USA; 2010.

19.- Baffone W, Citterio B, Vittoria E. Determination of several potential virulence factors in Vibrio spp. isolated from sea water. Food Microbiol 2001; 18: 479-88.

20.- Hammer O, Harper D A T, Ryan P D. PAST: Palenteological statistics software package for education and data analysis. Palentologia Electronica 2001; 4: 9.

21.- Dirección General de Epidemiología (DGE). Anuario de Morbilidad 1984-2014. Morbilidad nacional. http://www.epidemiologia.salud.gob. $\mathrm{mx} /$ anuario/html/morbilidad_enfermedad.html (Accedido el 16 de febrero de 2016).

22.- Domínguez C, Gómez I, Zumalacárregui J. Prevalence of Salmonella and Campylobacter in retail chicken meat in Spain. Int J Food Microbiol 2002; 72: 165-8.

23.- Chen M H, Wang S W, Hwang W Z, Tsai S J, Hsih Y C, Chiou Y C, et al. Contamination of Salmonella Schwarzengrund cells in chicken meat from traditional marketplaces in Taiwan and comparison of their antibiograms with those of the human isolates. Poult Sci 2010; 89: 359-65.

24.- Fearnley E, Raupach J, Lagala F, Cameron S. Salmonella in chicken meat, eggs and humans; Adelaide, South Australia, 2008. Int J Food Microbiol 2011; 146: 219-27.

25.- El-Aziz D M. Detection of Salmonella Typhimurium in retail chicken meat and chicken giblets. Asian Pac J Trop Biomed 2013; 3: 678-81.

26.- Khalid I S, Mohammed M A, Hassan M A, Tamura T. Prevalence, molecular identification and antimicrobial resistance profile of Salmonella serovars isolated from retail beef products in Mansoura, Egypt. Food Control 2014; 38: 209-14

27.- Jarquin C, Álvarez D, Morales O, Morales A J, López B, Donado P. et al. Salmonella on raw poultry in retail markets in Guatemala: Levels, antibiotic susceptibility, and serovar distribution. J Food Prot 2015; 9: 1642-50.

28.- Tadesse G, Tessema T S. A meta-analysis of the prevalence of Salmonella in food animals in Ethiopia. BMC Microbiology 2014; 14: 270.

29.- Jordan E, Egan J, Dullea C, Ward J, McGillicuddy K, Murray G, et al. Salmonella surveillance in raw and cooked meat and meat products in the Republic of Ireland from 2002 to 2004. Int J Food Microbiol 2006; 112: 66-70.

30.- Little C L, Richardson J F, Owen R J, De Pinna E, Threlfall E J. Campylobacter and Salmonella in raw meats in the United Kingdom:

Prevalence, characterization and antimicrobial resistance pattern, 2003-2005. Food Microbiol 2008; 25: 538-43

31.- MacDowell S W, Porter R, Madden R, Cooper B, Neill S D. Salmonella in slaughter pigs in Northern Ireland: Prevalence and use of statistical modelling to investigate sample and abbattoir effects. Int J Food Microbiol 2007; 118: $116-25$

32.- Magwedere K, Rauff D, De Klerk G, Keddy $\mathrm{K}$ H, Dziva F. Incidence of nontyphoidal Salmonella in food-producing animals, animal feed, and the associated environment in South Africa, 2012-2014. Clin Infect Dis 2015; 61: 283-9.

33.- Foley S L, Lynne A M. Food animal-associated Salmonella challenges: Pathogenicity and antimicrobial food animal-associated. J Anim Sci 2008; 86: 173-87.

34.- Cabrera-Díaz E, Barbosa-Cárdenas C M, Pérez-Montaño J A, González-Aguilar D, Pacheco-Gallardo C, Barba J. Ocurrence, serotype diversity, and antimicrobial resistance of Salmonella in ground beef at retail stores in Jalisco State, Mexico. J Food Prot 2013; 76: 2004-10.

35.- Crandall P G, O’Bryan C A, Babu D, Jarvis $\mathrm{N}$, Davis M L, Buser M, et al. Whole-chain traceability, is it possible to trace your hamburger to a particular steer, a U. S. perspective. Meat Sci 2013; 95: 137-44.

36.- Martínez-Chávez L, Cabrera-Díaz E, PérezMontaño J A, Garay-Martínez L E, VarelaHernández J J, Castillo A, et al. Quantitative distribution of Salmonella spp. and Escherichia coli on beef carcasses and raw beef at retail establishments. Int J Food Microbiol 2015; 210 : 149-55. 
37.- Carrasco E, Morales-Rueda A, García-Gimeno R M. Cross-contamination and recontamination by Salmonella in foods: A review. Food Res Int 2012; 45: 545-56.

38.- Dirección General de Epidemiología (DGE). Anuario de Morbilidad 1984-2014. Incidencia de enfermedad por grupo de edad y entidad federativa. http://www.epidemiologia.salud. gob.mx/anuario/html/incidencia_enfermedad. html (Accedido el 16 de febrero de 2016).

39.- Gutiérrez-Cogco L, Montiel-Vázquez E, Aguilera-Pérez P, González-Andrade M C. Serotipos de Salmonella identificados en los servicios de salud de México. Salud Pública Mex 2000; 42: 490-5.

40.- Sibhat B, Molla-Zewde B, Zerihun A, Muckle A, Cole L, Boerlin P, et al. Salmonella serovars and antimicrobial resistance profiles in beef cattle, slaughterhouse personnel and slaughterhouse environment in Ethiopia. Zoonoses Public Health 2011; 58: 102-9.

41.- Secretaría de Agricultura, Ganadería, Desarrollo Rural, Pesca y Alimentación (SAGARPA). Diario Oficial de la Federación. Acuerdo por el que se modifica el diverso por el que se establece la clasificación y prescripción de los productos farmacéuticos veterinarios por el nivel de riesgo de sus ingredientes activos. http://www.facmed.unam.
mx/deptos/microbiologia/pdf/DIARIO $\% 20$ OFICIAL_05\%2003\%202012\%20 ACUERDO\%20farmaceuticos\%20veterinarios. pdf (Accedido el 28 de noviembre de 2016).

42.- Cardona-Castro N, Sánchez-Jiménez M. Evaluación de la capacidad de invasión de serovariedades de Salmonella enterica a células HEp-2. Rev CES Medicina 2005; 19: 7-16.

43.- Figueroa-Ochoa I, Verdugo-Rodríguez A. Mecanismos moleculares de patogenicidad de Salmonella sp. Rev Latinoam Microbiol 2005; 47: 25-42.

44.- Vidal J E, Canizález-Román A, GutiérrezJiménez J, Navarro-García F. Patogénesis molecular, epidemiología y diagnóstico de Escherichia coli enteropatógena. Salud Publica Mex 2007; 49: 376-86.

45.- Althouse C, Patterson S, Fedorka-Cray P, Isaacso R E. Type 1 fimbriae of Salmonella enterica serovar Typhimurium bind to enterocytes and contribute to colonization of swine in vivo. Infect Immun 2003; 71: 6446-52.

46.- Ledeboer N A, Frye J G, McClelland M, Jones B D. Salmonella enterica serovar Typhimurium requires the Lpf, Pef, and Tafi fimbriae for biofilm formation on HEp-2 tissue culture cells and chicken intestinal epithelium. Infect Immun 2006; 74: 3156-69.
47.- Dwyer B E, Newton K L, Kisiela D, Sokurenko E V, Clegg S. Single nucleotide polypmorphisms of fimH associated with adherence and biofilm formation by serovars of Salmonella enterica. Microbiology 2011; 157: 3162-71.

48.- Shu-Kee E, Pusparajah P, Nurul-Syakima A M, HooiLeng S, Kok-Gan Chan, Learn-Han L. Salmonella: A review on pathogenesis, epidemiology and antibiotic resistance. Frontiers in Life Science 2015; 8: 284-93.

49.- Chaudhuri R R, Morgan E, Peters S E, Pleasance S J, Hudson D L, Davies H M, et al. Comprehensive assignment of roles for Salmonella Typhimurium genes in intestinal colonization of food-producing animals. PLoS Genet 2013; 9: e1003456.

50.- Aarestrup F M, Hendriksen R S, Lockett J, Gay K, Teates K, McDermott, et al. International spread of multidrug-resistant Salmonella Schwarzengrund in food products. Emerg Infect Dis 2007; 13: 726-31.

51.- Bai L, Lan R, Zhang X, Cui S, Xu J, Guo Y, et al. Prevalence of Salmonella isolates from chicken and pig slaughterhouses and emergence of ciprofloxacin and cefotaxime co-resistant $S$. enterica serovar Indiana in Henan, China. PLoS ONE 2015; 10: $\mathrm{e} 0144532$. 